\title{
Existence and Uniqueness of Solution to Semilinear Fractional Elliptic Equation
}

\author{
Shangjian Liu \\ College of Science, University of Shanghai for Science and Technology, Shanghai, China \\ Email: lxjfyt@163.com
}

How to cite this paper: Liu, S.J. (2019) Existence and Uniqueness of Solution to Semilinear Fractional Elliptic Equation. Journal of Applied Mathematics and Physics, 7, 210-217. https://doi.org/10.4236/jamp.2019.71017

Received: January 10, 2019

Accepted: January 22, 2019

Published: January 25, 2019

Copyright $\odot 2019$ by author(s) and Scientific Research Publishing Inc. This work is licensed under the Creative Commons Attribution International License (CC BY 4.0).

http://creativecommons.org/licenses/by/4.0/

\section{Abstract}

In this work, we study the following problem.

$\left\{\begin{array}{ll}(-\Delta)^{s} u+g(u)=f(x), & x \in \Omega, \\ u=0, & x \in \mathbb{R}^{N} \backslash \Omega,\end{array}\right.$, where $(-\Delta)^{s}$ is the fractional Laplacian and $\Omega$ is a bounded domain in $\mathbb{R}^{N}$ with Lipschitz boundary. $g: \mathbb{R} \rightarrow \mathbb{R}$ is an increasing locally Lipschitz continuous function. and $f \in L^{m}(\Omega), m \geq \frac{2 N}{N+2 s}$. We use Stampacchia's theorem to study existence of the solution $u$, and we prove the uniqueness of $u$ by contradiction.

\section{Keywords}

Sobolev Embedding Theorem, Stampacchia's Theorem, Existence, Uniquness

\section{Introduction}

In recent years, many people pay attention to the fractional Laplacian. One of the reasons for this comes from the fact that this operator naturally arises in several phenomena like flames propagation and geophysical fluid dynamics, or in mathematical finance. About the Fractional Sobolev space we can refer [1] [2]. In this work, we consider the problem

$$
\begin{cases}(-\Delta)^{s} u+g(u)=f(x), & x \in \Omega, \\ u=0, & x \in \mathbb{R}^{N} \backslash \Omega .\end{cases}
$$

where $s \in(0,1), N>2 s, \Omega \in \mathbb{R}^{N}$ is a bounded domain with Lipschitz boundary. $(-\Delta)^{s}$ as the fractional Laplacian, which defined as

$$
(-\Delta)^{s} u(x)=a_{N, s} P \cdot V \cdot \int_{\mathbb{R}^{N}} \frac{u(x)-u(y)}{|x-y|^{N+2 s}} \mathrm{~d} y,
$$


where

$$
a_{N, s}=\left(\int_{\mathbb{R}^{N}} \frac{1-\cos \left(\xi_{1}\right)}{\left|\xi_{1}\right|^{N+2 s}}\right)^{-1}=2^{2 s-1} \pi^{-\frac{N}{2}} \frac{\Gamma\left(\frac{N+2 s}{2}\right)}{|\Gamma(-s)|}
$$

It is worthy to point out that

$$
\lim _{s \rightarrow 0} a_{N, s}=0=\lim _{s \rightarrow 1} a_{N, s},
$$

we can refer [3].

For $s \in(0,1)$, we can also define the fractional Laplacian $(-\Delta)^{s}$ as the operator given by the Fourier multiplier $|\xi|^{2 s}$, that is, for $u \in S\left(\mathbb{R}^{N}\right)$

$$
\mathcal{F}\left((-\Delta)^{s} u\right)(\xi)=|\xi|^{2 s} \mathcal{F}(u)(\xi),
$$

where we denote by $S\left(\mathbb{R}^{N}\right)$ the class of all Schwartz functions in $\mathbb{R}^{N}$.

We introduce the Sobolev space

$$
H^{s}\left(\mathbb{R}^{N}\right)=\left\{u \in L^{2}\left(\mathbb{R}^{N}\right):|\xi|^{s} \mathcal{F}(u)(\xi) \in L^{2}\left(\mathbb{R}^{N}\right)\right\},
$$

and the space $H_{0}^{s}(\Omega)$

$$
H_{0}^{s}(\Omega)=\left\{u \in H^{s}(\Omega), u=0 \text { a.e. } x \in \mathbb{R}^{N} \backslash \Omega\right\},
$$

endowed with the norm

$$
\|u\|_{H_{0}^{s}(\Omega)}=\left(\iint_{D_{\Omega}} \frac{|u(x)-u(y)|^{2}}{|x-y|^{N+2 s}} \mathrm{~d} x \mathrm{~d} y\right)^{\frac{1}{2}},
$$

where $D_{\Omega}=\mathbb{R}^{N} \times \mathbb{R}^{N} \backslash \mathcal{C} \Omega \times \mathcal{C} \Omega, C \Omega=\mathbb{R}^{N} \backslash \Omega$. This space allows us to deal with the problems proposed in a bounded domain $\Omega$, as we need. The pair $\left(H_{0}^{s}(\Omega),\|\cdot\|_{H_{0}^{s}(\Omega)}\right)$ yields a Hilbert space [4]. Moreover, it can be seen that

$$
(-\Delta)^{s}: H_{0}^{s}(\Omega) \rightarrow H^{-s}(\Omega)
$$

is a continuous operator.

Theorem 1.1. Let $g: \mathbb{R} \rightarrow \mathbb{R}$ be an increasing locally Lipschitz continuous function. Let $f \in L^{m}(\Omega), m \geq \frac{2 N}{N+2 s}$. Then (1.1) have a unique solution $u \in H_{0}^{s}(\Omega)$. Moreover,

$$
g(u) \in L^{1}(\Omega)
$$

\section{Preliminaries}

In this section, we give some basic results of fractional Sobolev space $H_{0}^{s}(\Omega)$ that will be used in the next section.

Definition 2.1 We say that $u \in H_{0}^{s}(\Omega)$ is a weak solution to (1.1) if we have

$$
\iint_{D_{\Omega}} \frac{(u(x)-u(y))(\varphi(x)-\varphi(y))}{|x-y|^{N+2 s}} \mathrm{~d} x \mathrm{~d} y+\int_{\Omega} g(u) \varphi \mathrm{d} x=\int_{\Omega} f \varphi \mathrm{d} x,
$$


for any $\varphi \in H_{0}^{s}(\Omega) \cap L^{\infty}(\Omega)$.

Lemma 2.1. [5] Let $N \geq 1$ and $s \in(0,1)$.Then for all $u \in H^{s}(\Omega)$ we have

$$
\int_{\mathbb{R}^{N}}|\xi|^{2 s}|\mathcal{F}(u)(\xi)|^{2} \mathrm{~d} \xi=a_{N, s} \iint_{\mathbb{R}^{N} \times \mathbb{R}^{N}} \frac{|u(x)-u(y)|^{2}}{|x-y|^{N+2 s}} \mathrm{~d} x \mathrm{~d} y,
$$

where $a_{N, s}$ is the constant defined in (1.3).

Proof. Fixed $y$ we change coordinates $z=x-y$ and apply Plancherel.

Recalling that $(u(\cdot+z))^{\wedge}(\xi)=\mathrm{e}^{i \xi \cdot z} \hat{u}(\xi)$ we obtain

$$
\iint \frac{|u(x)-u(y)|^{2}}{|x-y|^{N+2 s}} \mathrm{~d} x \mathrm{~d} y=\int\left(\int|z|^{-(N+2 s)}\left|\mathrm{e}^{i \xi \cdot z}-1\right|^{2} \mathrm{~d} z\right)|\hat{u}(\xi)|^{2} \mathrm{~d} \xi
$$

The integral in brackets is of the form $c_{N, s}|\xi|^{2 s}$, with

$$
\begin{aligned}
c_{N, s} & :=\int_{0}^{\infty} \int_{\mathcal{S}^{N-1}}\left|\mathrm{e}^{i r \omega \cdot \theta}-1\right|^{2} \mathrm{~d} \theta r^{-2 s-1} \mathrm{~d} r \\
& =2 \int_{0}^{\infty}\left(\left|\mathcal{S}^{N-1}\right|-(2 \pi)^{N / 2} r^{-(N-2) / 2} J_{(N-2) / 2}(r)\right) r^{-(2 s+1)} \mathrm{d} r
\end{aligned}
$$

where $J_{(N-2) / 2}$ is the Bessel function of the first kind of order $(N-2) / 2$, we can refer [6].

Recall that $\left|\mathcal{S}^{N-1}\right|=2 \pi^{N / 2} / \Gamma(N / 2)$. The formula (1.3) for $c_{N, s}=a_{N, s}^{-1}$ now follows from

$$
\int_{0}^{\infty} r^{-z}\left(J_{(N-2) / 2}(r)-2^{-(N-2) / 2} \Gamma(N / 2)^{-1} r^{(N-2) / 2}\right) \mathrm{d} r=2^{-z} \frac{\Gamma((N-2 z) / 4)}{\Gamma((N+2 z) / 4)},
$$

for $N / 2<\operatorname{Re} z<(N+4) / 2$, we can see[5].

Lemma 2.2. [7] For $s \in(0,1), N \geq 2 s$, there exists a positive constant $C=C(N, s)$, for any $u \in D^{s}\left(\mathbb{R}^{N}\right)$,we have

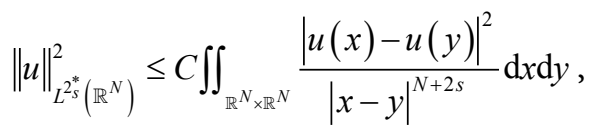

where $2_{s}^{*}=\frac{2 N}{N-2 s}$ is called fractional critical Sobolev exponent. In particular, if $u \in H_{0}^{s}(\Omega)$ then

$$
\|u\|_{L^{*}(\Omega)}^{2} \leq C \iint_{D_{\Omega}} \frac{|u(x)-u(y)|^{2}}{|x-y|^{N+2 s}} \mathrm{~d} x \mathrm{~d} y .
$$

Lemma 2.3. (Egorov's theorem) [8] Let $f_{n}$ be a sequence of functions and $f$ be a function defined on $E$, with meas $(E)<+\infty$. Assume that $f_{n} \rightarrow f$ a.e. in $E$. Then for every $\varepsilon>0$ there exists a measurable subset $A$ of $E$ such that meas $(E \backslash A)<\varepsilon$ and $f_{n} \rightarrow f$ uniformly on $A$, as $n \rightarrow \infty$.

Lemma 2.4. (Vitali) [9] Let $f_{n}$ be a sequence of functions and $f$ be a function in $L^{p}(\Omega)$. Assume that

1) $f_{n} \rightarrow f$ a.e. in $\Omega$;

2) if $E$ is a measurable subset of $\Omega$, and we have

$$
\lim _{\text {meas }(E) \rightarrow 0} \int_{E}\left|f_{n}\right|^{p}=0 \text {, }
$$


uniformly with respect $n$. Where meas $(E)$ means measure representing $E$. Then $f_{n} \rightarrow f$ in $L^{p}(\Omega)$.

Proof. Fixed $\varepsilon>0$, let $E \subset \Omega$ be a measurable set, we have

$$
\int_{\Omega}\left|f_{n}-f\right|^{p} \leq \int_{\mathbb{R}^{\mathcal{N}} \backslash \Omega}\left|f_{n}-f\right|^{p}+2^{p-1} \int_{E}\left(\left|f_{n}\right|^{p}+|f|^{p}\right) .
$$

Using assumption (2), we know that there exists $\delta_{1}(\varepsilon)>0$ such that, if meas $(E)<\delta_{1}(\varepsilon)$, then for any $n \in \mathbb{N}$ we have

$$
\int_{E}\left|f_{n}\right|^{p}<\varepsilon
$$

Since $f \in L^{p}(\Omega)$ there exists $\delta_{2}(\varepsilon)>0$ such that if meas $(E)<\delta_{2}(\varepsilon)$, then

$$
\int_{E}|f|^{p}<\varepsilon
$$

In conclusion the second term of the right-hand side of (2.18) is less than $2^{p} \varepsilon$. Let us study the first one. We set $\delta=\min \left\{\delta_{1}(\varepsilon), \delta_{2}(\varepsilon)\right\}$, and use Egorov's theorem, there exist $v_{\varepsilon} \in \mathbb{N}$ and a measurable set $E_{0} \subset \Omega$ such that $\operatorname{meas}\left(E_{0}\right)<\delta$, and

$$
\int_{\Omega E_{0}}\left|f_{n}-f\right|^{p}<\varepsilon,
$$

for any $n>v_{\varepsilon}$.Choosing $E=E_{0}$ in(2.18), we get the result.

Lemma 2.5. (Stampacchia) [10] Let $H$ be a Hilbert space, $a: H \times H \rightarrow \mathbb{R}$ is a continuous and linear form in the second variable such that

1) for $\beta \in \mathbb{R}^{+}$,any $\psi_{1}, \psi_{2}, w \in H$, we have

$$
\left|a\left(\psi_{1}, w\right)-a\left(\psi_{2}, w\right)\right| \leq \beta\left\|\psi_{1}-\psi_{2} \mid\right\| w \|,
$$

2) for a positive constant $C$, any $\psi_{1}, \psi_{2} \in H$ we have

$$
a\left(\psi_{1}, \psi_{1}-\psi_{2}\right)-a\left(\psi_{2}, \psi_{1}-\psi_{2}\right) \geq C\left\|\psi_{1}-\psi_{2}\right\|^{2} .
$$

Lemma 2.6. (Hölder inequality) [11] Let $p$ and $q$ are dual indicators, stisfies

$$
1 / p+1 / q=1
$$

where $1 \leq p \leq \infty$, if $f \in L^{p}(\Omega)$, and $g \in L^{q}(\Omega)$, then the product of $(f g)(x)=f(x) g(x)$ the defined function belongs to $L^{1}(\Omega)$, and we have

$$
\left|\int_{\Omega} f g \mathrm{~d} x\right| \leq \int_{\Omega}|f||g| \mathrm{d} x \leq\|f\|_{p}\|g\|_{q} .
$$

If and only if there is a real constant $m$ that makes the following formula hold

$$
f g=\mathrm{e}^{i m}|f||g| \text {. }
$$

The first unequal sign of (2.24) is established. If $f$ not constant equals 0 , then the second unequal sign of (2.24) is established, if and only if there exists a constant $\eta \in \mathbb{R}$, such that

1) if $1<p<\infty$, then $|g(x)|=\eta|f(x)|^{p-1} \mu$ a.e. $\in \Omega$.

2) if $p=1$, then $|g(x)| \leq \eta \quad \mu$ a.e. $\in \Omega$, and when $f(x) \neq 0$, we have 
$|g(x)|=\eta$.

3) if $p=\infty$, then $|f(x)| \leq \eta \quad \mu$ a.e. $\in \Omega$, and when $g(x) \neq 0$, we have $|f(x)|=\eta$.

\section{Proof of Theorem 1.1}

Theorem 3.1. Let $g: \mathbb{R} \rightarrow \mathbb{R}$ be an increasing function, and $g$ is Lipschitz continuous, that is, there exists a positive constant $\mu$ such that for any $s, t \in \mathbb{R}$ we have

$$
|g(s)-g(t)| \leq \mu|s-t|,
$$

Let $f \in L^{m}(\Omega), m \geq \frac{2 N}{N+2 s}$. Then (1.1) exists a unique solution $u \in H_{0}^{s}(\Omega)$.

Proof. We define the following form on $H_{0}^{s}(\Omega) \times H_{0}^{s}(\Omega)$ :

$$
a(u, w)=\iint_{D_{\Omega}} \frac{(u(x)-u(y))(w(x)-w(y))}{|x-y|^{N+2 s}} \mathrm{~d} x \mathrm{~d} y+\int_{\Omega} g(u) w \mathrm{~d} x .
$$

Using Hölder inequality and (3.1) we have

$$
|a(u, w)| \leq \iint_{D_{\Omega}} \frac{u(x)-u(y)}{|x-y|^{\frac{N+2 s}{2}}} \cdot \frac{w(x)-w(y)}{|x-y|^{\frac{N+2 s}{2}}} \mathrm{~d} x \mathrm{~d} y+\int_{\Omega}[\mu|u|+g(0)]|w| \mathrm{d} x,
$$

that is, $a$ is well defined. By the definition of $a$, we know that $a$ is continuous and linear in the second variable. If $w_{n} \rightarrow w$ in $H_{0}^{s}(\Omega)$, then

$$
\begin{gathered}
\iint_{D_{\Omega}} \frac{(u(x)-u(y))\left(w_{n}(x)-w_{n}(y)\right)}{|x-y|^{N+2 s}} \mathrm{~d} x \mathrm{~d} y \\
\rightarrow \iint_{D_{\Omega}} \frac{(u(x)-u(y))(w(x)-w(y))}{|x-y|^{N+2 s}} \mathrm{~d} x \mathrm{~d} y \\
\int_{\Omega} g(u) w_{n} \mathrm{~d} x \rightarrow \int_{\Omega} g(u) w \mathrm{~d} x .
\end{gathered}
$$

Since

$$
\begin{aligned}
& \left|a\left(u_{1}, w\right)-a\left(u_{2}, w\right)\right| \\
& =\mid \iint_{D_{\Omega}} \frac{\left(u_{1}(x)-u_{1}(y)\right)(w(x)-w(y))}{|x-y|^{N+2 s}} \mathrm{~d} x \mathrm{~d} y \\
& \quad-\iint_{D_{\Omega}} \frac{\left(u_{2}(x)-u_{2}(y)\right)(w(x)-w(y))}{|x-y|^{N+2 s}} \mathrm{~d} x \mathrm{~d} y|+| \int_{\Omega}\left[g\left(u_{1}\right)-g\left(u_{2}\right)\right] w \mathrm{~d} x \mid \\
& =\left|\iint_{D_{\Omega}} \frac{\left(u_{1}-u_{2}\right)(x)-\left(u_{1}-u_{2}\right)(y)}{|x-y|^{\frac{N+2 s}{2}}} \cdot \frac{\left(w_{1}\right)(x)-\left(w_{2}\right)(y)}{|x-y|^{\frac{N+2 s}{2}}}\right|+\left|\int_{\Omega}\left[g\left(u_{1}\right)-g\left(u_{2}\right)\right] w \mathrm{~d} x\right| \\
& \leq\left\|u_{1}-u_{2}\right\|_{H_{0}^{s}(\Omega)}|| w\left\|_{H_{0}^{s}(\Omega)}+\mu\right\| u_{1}-u_{2}\left\|_{L^{2}(\Omega)}\right\| w \|_{L^{2}(\Omega)},
\end{aligned}
$$

the last inequality following from Hölder inequality and (3.1), by lemma 2.2

$$
\left|a\left(u_{1}, w\right)-a\left(u_{2}, w\right)\right| \leq\left(1+\mu C^{2}\right)\left\|u_{1}-u_{2}\right\|_{H_{0}^{s}(\Omega)}\|w\|_{H_{0}^{s}(\Omega)} .
$$


Since

$$
\begin{aligned}
& a\left(u_{1}, u_{1}-u_{2}\right)-a\left(u_{2}, u_{1}-u_{2}\right) \\
& =\iint_{D_{\Omega}} \frac{\left|\left(u_{1}-u_{2}\right)(x)-\left(u_{1}-u_{2}\right)(y)\right|^{2}}{|x-y|^{N+2 s}} \mathrm{~d} x \mathrm{~d} y+\int_{\Omega}\left[g\left(u_{1}\right)-g\left(u_{2}\right)\right]\left(u_{1}-u_{2}\right) \mathrm{d} x
\end{aligned}
$$

by (3.1)

$$
\int_{\Omega}\left[g\left(u_{1}\right)-g\left(u_{2}\right)\right]\left(u_{1}-u_{2}\right) \mathrm{d} x \geq 0
$$

then

$$
a\left(u_{1}, u_{1}-u_{2}\right)-a\left(u_{2}, u_{1}-u_{2}\right) \geq\left\|u_{1}-u_{2}\right\|_{H_{0}^{s}(\Omega)}^{2} .
$$

We know that a satisfies lemma 2.4 from (3.2) and (3.10), the result follows from lemma 2.4.

We define the following function, for $k>0$ :

$$
T_{k}(s)=\left\{\begin{array}{lc}
-k, & s \leq-k, \\
s, & |s| \leq k, \\
k, & s \geq k .
\end{array}\right.
$$

Proof of theorem 1.1: First, we proof the existence of a solution by approximation. Let $g_{n}(t)=T_{n}(g(t))$, By theorem 3.1 we know that there exists $u_{n} \in H_{0}^{s}(\Omega)$ be the solution to problems

$$
\begin{cases}(-\Delta)^{s} u_{n}+g_{n}\left(u_{n}\right)=f(x), & x \in \Omega, \\ u_{n}=0, & x \in \mathbb{R}^{N} \backslash \Omega .\end{cases}
$$

We use $u_{n}$ as a test function in (3.12), we get

$$
\left\|u_{n}\right\|_{H_{0}^{s}(\Omega)}^{2}+\int_{\Omega} u_{n} g_{n}\left(u_{n}\right) \mathrm{d} x=\int_{\Omega} f u_{n} \mathrm{~d} x .
$$

Then use Hölder inequality on the right-hand side implies

$$
\begin{aligned}
& \left\|u_{n}\right\|_{H_{0}^{s}(\Omega)}^{2}+\int_{\Omega} u_{n} g_{n}\left(u_{n}\right) \mathrm{d} x \\
& \leq\left(\int_{\Omega} f^{\frac{2 N}{N+2 s}} \mathrm{~d} x\right)^{\frac{N+2 s}{2 N}}\left(\int_{\Omega} u_{n}^{2_{s}^{*}} \mathrm{~d} x\right)^{\frac{1}{2_{s}^{*}}}=\|f\|_{L^{\frac{2 N}{N+2 s}}(\Omega)}\left\|u_{n}\right\|_{L^{2_{s}^{*}}(\Omega)} .
\end{aligned}
$$

Because $g$ is increasing, then $\left\|u_{n}\right\|_{H_{0}^{s}(\Omega)}^{2} \leq\|f\|_{L^{N+2 s}(\Omega)}\left\|u_{n}\right\|_{L^{2 *}(\Omega)}$. This means $\left\|u_{n}\right\|_{H_{0}^{s}(\Omega)}$ is uniformly bounded. We can deduce there exists $u_{n} \rightarrow u$ weakly in $H_{0}^{s}(\Omega)$ and a.e., since $\left\|u_{n}\right\|_{H_{0}^{s}(\Omega)}^{2} \geq 0$, by (3.13) there exists a positive constant $C$ such that

$$
\int_{\Omega} u_{n} g_{n}\left(u_{n}\right) \mathrm{d} x \leq C
$$

for every $n$.

Now we prove $g_{n}\left(u_{n}\right) \rightarrow g(u)$ in $L^{1}(\Omega)$. Since $g$ is continuous in $\Omega$ then it is clear that $g_{n}\left(u_{n}\right) \rightarrow g(u)$ a.e. in $\Omega$. If $E$ is a subset of $\Omega$, for $t \in \mathbb{R}^{+}$ have 


$$
\begin{aligned}
\int_{E}\left|g_{n}\left(u_{n}\right)\right| & =\int_{\left\{x \in E:\left|u_{n}(x) \leq t\right|\right\}}\left|g_{n}\left(u_{n}\right)\right|+\int_{\left\{x \in E: u_{n}(x)>t \mid\right\}}\left|g_{n}\left(u_{n}\right)\right| \\
& \leq \int_{E}\left|g_{n}(t)\right|+\frac{1}{t} \int_{\left\{x \in E:\left|u_{n}(x)>t\right|\right\}} u_{n} g\left(u_{n}\right) \\
& \leq|g(t)| \text { meas }(E)+\frac{C}{t},
\end{aligned}
$$

combining (3.14), for $t \in \mathbb{R}^{+}$we have

$$
\lim _{\operatorname{meas}(E) \rightarrow 0} \int_{E}\left|g_{n}\left(u_{n}\right)\right| \leq \frac{C}{t} .
$$

Using lemma 2.4, we know that $g_{n}\left(u_{n}\right) \rightarrow g(u)$ in $L^{1}(\Omega)$. Then for any $\phi \in H_{0}^{s}(\Omega) \cap L^{\infty}(\Omega)$

we from

$$
\iint_{D_{\Omega}} \frac{\left(u_{n}(x)-u_{n}(y)\right)(\phi(x)-\phi(y))}{|x-y|^{N+2 s}} \mathrm{~d} x \mathrm{~d} y+\int_{\Omega} g_{n}\left(u_{n}\right) \phi \mathrm{d} x=\int_{\Omega} f \phi \mathrm{d} x
$$

get

$$
\iint_{D_{\Omega}} \frac{(u(x)-u(y))(\phi(x)-\phi(y))}{|x-y|^{N+2 s}} \mathrm{~d} x \mathrm{~d} y+\int_{\Omega} g(u) \phi \mathrm{d} x=\int_{\Omega} f \phi \mathrm{d} x .
$$

Finally we prove the solution of problem (1.1) is unique. We assume $u_{1}$ and $u_{2}$ are two solutions, $u_{1} \neq u_{2}$, we take $u_{1}-u_{2}$ as a test function

$\iint_{D_{\Omega}} \frac{\left(u_{1}(x)-u_{1}(y)\right)\left[\left(u_{1}-u_{2}\right)(x)-\left(u_{1}-u_{2}\right)(y)\right]}{|x-y|^{N+2 s}} \mathrm{~d} x \mathrm{~d} y+\int_{\Omega} g\left(u_{1}\right)\left(u_{1}-u_{2}\right) \mathrm{d} x$

$=\int_{\Omega} f\left(u_{1}-u_{2}\right) \mathrm{d} x$,

$\iint_{D_{\Omega}} \frac{\left(u_{2}(x)-u_{2}(y)\right)\left[\left(u_{1}-u_{2}\right)(x)-\left(u_{1}-u_{2}\right)(y)\right]}{|x-y|^{N+2 s}} \mathrm{~d} x \mathrm{~d} y+\int_{\Omega} g\left(u_{2}\right)\left(u_{1}-u_{2}\right) \mathrm{d} x$

$=\int_{\Omega} f\left(u_{1}-u_{2}\right) \mathrm{d} x$.

We can deduce from (3.19) and (3.20)

$$
\begin{aligned}
& \iint_{D_{\Omega}} \frac{\left[u_{1}(x)-u_{1}(y)-u_{2}(x)+u_{2}(y)\right]\left[\left(u_{1}-u_{2}\right)(x)-\left(u_{1}-u_{2}\right)(y)\right]}{|x-y|^{N+2 s}} \mathrm{~d} x \mathrm{~d} y \\
& =\int_{\Omega}\left(g\left(u_{1}\right)-g\left(u_{2}\right)\right)\left(u_{1}-u_{2}\right) \mathrm{d} x .
\end{aligned}
$$

This means

$$
\left\|u_{1}-u_{2}\right\|_{H_{0}^{s}(\Omega)}^{2}=\int_{\Omega}\left(g\left(u_{1}\right)-g\left(u_{2}\right)\right)\left(u_{1}-u_{2}\right) \mathrm{d} x .
$$

By the monotonicity of $g$ we know

$$
\int_{\Omega}\left(g\left(u_{1}\right)-g\left(u_{2}\right)\right)\left(u_{1}-u_{2}\right) \mathrm{d} x \leq 0 .
$$

Combining (3.22) and (3.23) we know $u_{1}=u_{2}$ a.e. in $\Omega$.

\section{Conflicts of Interest}

The author declares no conflicts of interest regarding the publication of this pa- 
per.

\section{References}

[1] Tommaso, L., Ireneo, P., Ana, P. and Fernando, S. (2015) Basic Estimates for Solutions of a Class of Nonlocal Elliptic and Parabolic Equations. Discrete and Continuous Dynamical System, 35, 6031-6068.

[2] Begoña, B., Bonis, I.D. and Medina, M. (2015) Semilinear Problems for the Fractional Laplacian with a Singular Nonlinearity. Open Mathematics, 13.

[3] Nezza, E.D., Palatucci, G. and Valdinoci, E. (2012) Hitchhiker's Guide to the Fractional Sobolev Spaces. Bulletin Des Sciences Mathmatiques, 136, 521-573.

https://doi.org/10.1016/j.bulsci.2011.12.004

[4] Servadei, R. and Valdinoci, E. (2012) Mountain Pass Solutions for Non-Local Elliptic Operators. Journal of Mathematical Analysis \& Applications, 389, 887-898. https://doi.org/10.1016/j.jmaa.2011.12.032

[5] Lieb, E.H. (1983) Sharp Constants in the Hardy-Littlewood-Sobolev and Related Inequalities. Annals of Mathematics, 118, 349-374. https://doi.org/10.2307/2007032

[6] Abramowitz, M., Stegun, I.A. and Miller, D. (1965) Handbook of Mathematical Functions with Formulas, Graphs and Mathematical Tables (National Bureau of Standards Applied Mathematics Series No. 55). Journal of Applied Mechanics, 32, 239. https://doi.org/10.1115/1.3625776

[7] Maz's, V. (2011) Sobolev Spaces with Applications to Elliptic Partial Differential Equations. 2nd Edition, Grundlehren der Mathematischen Wissenschaften, Spring, Heidelberg, 342.

[8] Boccardo, L. (2013) Elliptic Partial Differential Equations (Existence and Regularity of Distributional Solutions). Preliminaries of Real Analysis.

[9] Yafaev, D. (1999) Sharp Constants in the Hardy-Rellich Inequalities. Journal of Functional Analysis, 168, 121-144. https://doi.org/10.1006/jfan.1999.3462

[10] Boccardo, L. (2013) Elliptic Partial Differential Equations (Existence and Regularity of Distributional Solutions). Linear and Semilinear Elliptic Equations.

[11] Lieb, E.H. and Loss, M. (2001) Analysis. Graduate Studies in Mathematics. 